\title{
Dons anonymes et rencontres urbaines
}

Faire circuler des objets en ville

\author{
Valérie Guillard et Dominique Roux*
}

Les marchés de redistribution - ou de remise en circulation d'objets usagés - sont, avec les plateformes de fournitures de services et les systèmes d'échanges collaboratifs, l'un des trois domaines dans lequel se déploie l'économie collaborative. L'essor de ces nouvelles formes de redistribution est souvent associé à l'usage d'Internet, présenté comme l'un des supports essentiels de la fluidification des transactions. Comme l'expliquent Rachel Botsman et Roo Rogers dans leur ouvrage pionnier ${ }^{1}$ : «à l'ère pré-internet, les coûts de transactions nécessaires à la coordination des désirs et des besoins des individus, ou même seulement de leurs intérêts similaires étaient élevés, rendant le partage des produits et des marchandises délicat et peu pratique ». Les nouvelles plateformes d'échanges de biens usagés sur lesquels medias et universitaires braquent désormais le regard sont donc censées avoir permis d'atteindre une masse critique qui faisait défaut aux transactions locales. Cette focalisation sur la dimension numérique de la nouvelle économie du partage a pour effet de ravaler au rang d'archaïsme les façons plus traditionnelles d'échanger entre soi.

La consommation collaborative se réduit-elle pour autant à ces seuls collectifs intermédiés via Internet? Une bonne raison de répondre par la négative réside dans le fait qu'une grande partie des modes de circulation des biens est traditionnellement enracinée dans la sphère privée, dans des systèmes de relations personnelles et dans des formes locales d'échanges. Pour preuve, les résultats d'une récente étude des pratiques de consommation émergentes ${ }^{2}$ montrent que le troc se pratique davantage entre proches $(65 \%)$ que via un site Internet $(37 \%)$. Alors que les sites de troc et de don sont apparus sur Internet pour faciliter la circulation gratuite d'objets de seconde main, le « tout » virtuel dans lequel se développent les pratiques de consommation collaborative ne seraitil qu'une réalité surmédiatisée ? C'est un fait que les études récentes tendent à renforcer en se focalisant majoritairement sur ces «nouvelles» pratiques d'échanges. En contraignant leurs répondants à déclarer des comportements prédéfinis et mutuellement exclusifs comme revendre, donner, échanger ou troquer ${ }^{3}$, elles participent également à produire une vision cloisonnée de pratiques qui, en réalité, sont plus complexes et étroitement imbriquées dans la vie sociale.

Une autre explication possible du décalage entre la réalité des pratiques d'échange ou de don d'objets entre particuliers et l'excès d'attention portée aux réseaux virtuels tient à leurs limites, soulignées par quelques recherches récentes ${ }^{4}$. Ces limites sont tout d'abord liées à des

\footnotetext{
${ }^{1}$ Botsman R. et Roger R. (2010), What's mine is yours. The rise of collaborative consumption, Londres, Harper Collins, p. 126.

${ }^{2}$ ObSoCo (2013), L’Observatoire des consommations émergentes, Évolution des tendances de consommation. Rapport d'étude 2ème vague, Observatoire Société et consommation, Paris, octobre.

${ }^{3}$ Van De Walle I., Hebel P., Siounandan N. (2012), Les secondes vies des objets : les pratiques d'acquisition et de délaissement des produits de consommation, Cahier de recherche du Crédoc, $\mathrm{n}^{\circ} 290$, janvier.

${ }^{4}$ Guillard V. et Del Bucchia C. (2012), How about giving my things away over the Internet? When Internet makes it easier to give things away, Research in Consumer Behavior, 14, 47-65 ; Harvey J., Golightly D., Smith
} 
contraintes matérielles comme le temps et les efforts nécessaires pour décrire les produits à donner ou échanger, poster des annonces en ligne et attendre la réponse d'un possible interlocuteur. Elles sont également d'ordre cognitif, l'usage d'Internet n'étant pas nécessairement accessible ni familier à chacun, notamment lorsqu'il s'agit de l'utiliser à des fins de transactions ou d'échanges. Ces limites sont également physiques, car certains objets lourds et encombrants nécessitent que la passation des objets ait lieu au domicile de ceux qui les cèdent. Cette contrainte entraîne d'autres limites, sociales cette fois, liées à la rencontre. Celle-ci peut occasionner un embarras à voir autrui pénétrer dans sa sphère intime. En amont, sélectionner le «bon» destinataire auquel donner l'objet soulève également des arbitrages et questionnements d'ordre psychologique - à qui donner? Sur quel(s) critère(s)? Toutes ces raisons prises ensemble expliquent donc pourquoi les formes de troc et de don qui préexistaient au développement des plateformes informatisées ne disparaissent pas.

Rendre compte de l'épaisseur des pratiques collaboratives au-delà de l'iceberg numérique autour duquel se cristallise leur image nécessite donc de regarder finement par quelles modalités les objets circulent aussi en dehors de ces plateformes. C'est en examinant plus attentivement les initiatives individuelles et collectives qui facilitent cette circulation qu'il est possible de faire surgir des formes de collaboration urbaine inédites.

\section{Abandon et don d'objets}

Il est sans doute difficile d'imaginer qu'une économie collaborative puisse se mettre en place dans l'espace public, sur le trottoir, au ras du pavé. Pourtant, c'est bien le cas. La question est: comment? La réponse est à rechercher dans le système de collecte et de ramassage d'objets dits « encombrants» par les municipalités. Bien qu'il ne soit pas conçu pour être un espace de don entre ceux qui les déposent et ceux qui les ramassent, ce système permet de facto aux objets de circuler. Il offre en effet un moment, un espace et une opportunité de déposer - ou d'abandonner - des objets que d'autres peuvent récupérer.

Ceux que nous appellerons « les glaneurs » pour plus de commodité ont fait l'objet d'une attention récente des médias, toujours friands de nouveautés en matière d'évolutions des mœurs et de nouvelles pratiques. Emblématique de ces nouvelles formes d'approvisionnement qu'on tend à relier à la crise, le glanage perd pourtant peu à peu de son caractère marginal et anecdotique. Une étude conduite en $2013^{8}$ montre en effet que cette activité de ramassage d'objets dans l'espace public concerne désormais 40\% des Français dont 17\% le font parfois et $5 \%$ souvent. Toutefois, et en dépit du sens commun, l'étude souligne que la forte pénétration de cette pratique en milieu urbain et la diversité des profils qui s'y adonnent interdisent de la considérer comme uniquement associée à la grande pauvreté.

Les raisons pour lesquelles les glaneurs s'engagent dans cette pratique sont composites. Si la dimension économique lui semble traditionnellement attachée - comme le documentaire d'Agnès Varda (2000) Les Glaneurs et la glaneuse le montre en milieu rural -, d'autres motivations ont été mises en évidence ${ }^{9}$ Récupérer des objets obéit ainsi au désir de limiter le gaspillage, de prolonger la vie des objets, d'aider ceux qui, autour de soi, pourraient en avoir besoin. A côté de ces orientations écologiques, sociales et souvent critiques à l'égard de la société de consommation, glaner est aussi vécu par certains comme une activité ludique, une sorte de chasse

A. (2014), Giving and sharing in the digitally-mediated economy, Journal of Consumer Behaviour, à paraître, DOI: 10.1002/cb.1499 ; Nelson M.R. et Rademacher M.A. (2009), From trash to treasure: Freecycle.org as a case of generalized reciprocity, Advances in Consumer Research, 36, 905-906.

\footnotetext{
${ }^{8}$ ObSoCo (2013), op. cité.

${ }^{9}$ Brosius N., Fernandez K.V. et Cherrier H. (2012) Re-acquiring consumer waste: treasure in our trash?, Journal of Public Policy and Marketing, 32, 2, 1-53.
} 
au trésor dont les trouvailles sont par nature inattendues. Des motivations esthétiques président également à la récupération d'objets considérés comme déclassés ou démodés par ceux qui s'en débarrassent, tandis qu'ils font le bonheur des nostalgiques ou des amateurs avertis. Des régimes de goûts et de valeurs prêtées aux objets participent donc à rythmer leur circulation. Toutefois, l'attention portée aux motivations des glaneurs n'éclaire pas en quoi des formes de collaboration se font jour dans ces espaces urbains. Il faut pour cela comprendre symétriquement les comportements de ceux qui déposent leurs objets sur le trottoir et les intentions qui président à leurs choix ${ }^{10}$.

Pour ceux qui déposent des objets, le désir de se débarrasser coexiste souvent avec l'espoir qu'ils seront ramassés. En effet, pressés par l'urgence d'un déménagement, par la nécessité d'entreprendre des travaux ou par le désir de faire le vide chez eux, ils se trouvent à la fois soulagés de pouvoir évacuer aisément ce qui les encombre, mais parfois culpabilisés quant à la destruction prochaine d'objets qui pourraient encore resservir. Se résoudre à les poser sur le trottoir est donc une solution de facilité, sinon d'urgence, qui s'impose notamment lorsqu'il n'a pas été possible de donner les objets dans l'entourage proche ou lorsque ceux-ci ne répondent pas aux critères exigés par les organismes caritatifs. Les motivations à donner, plutôt qu'à jeter, sont clairement mises en avant par ceux qui déposent.

Toutefois, communiquer cette volonté est relativement difficile dans un système et dans un espace qui ne sont pas définis pour cela. Poser un objet sur le trottoir signifie au premier chef souhaiter s'en débarrasser et confier à la puissance publique le soin d'en assurer la collecte, la destruction ou le recyclage. Pour rendre plus manifeste ce désir de donner, certains enfreignent alors les règles et déposent leurs objets hors des moments prévus. Ils justifient leur pratique par le fait que ceux-ci disparaissent rapidement et, de fait, semblent intéresser des glaneurs. D'autres choisissent d'étiqueter les objets - «en état de marche », « je fonctionne encore », " prenez-moi » - et d'en faire les porte-parole de leur désir qu'ils resservent à d'autres. Parfois aussi, le moment pendant lequel les objets sont sortis du garage ou de la maison est l'occasion de constater qu'un passant est intéressé et de l'inviter explicitement à se servir.

Pour autant, ces rencontres sont rares et le désir de faire circuler les objets demeure à la merci du hasard. Aussi, certaines municipalités comme Amsterdam ont mis en place une solution permettant à ceux qui veulent donner, de déposer leurs objets sur le trottoir emballés dans un sac transparent. Ce «Goedzak » - qui signifie à la fois littéralement le «bon sac » et de «bon cœur »est un non seulement un dispositif qui protège les objets de la pollution, mais aussi un médiateur social qui atteste de leur (bon) état et de la volonté de son ancien propriétaire de leur donner une seconde vie.

Pour certaines catégories particulières de biens faciles à faire circuler, le système du « livre voyageur » ou Bookcrossing permet également de «relâcher» les livres déjà lus «dans la nature », c'est-à-dire dans n’importe quel endroit public en espérant que d'autres personnes les trouveront et les liront également. Parce qu'il fonctionne sur le même système d'emballage transparent des ouvrages et l'apposition de la marque de cette initiative, ce système organise la qualification et le repérage des objets abandonnés dans l'espace public par les membres de cette communauté de bibliophiles anonymes. S'il se prête bien à la circulation de produits de petite taille, le crossing est potentiellement déclinable sur d'autres catégories de biens. Mais il souffre de la double limite de prétendre embrasser tous les biens (et que la «libération » anarchique d'un trop grand nombre d'objets se mue rapidement en pollution de l'espace public. Aussi, corrigeant la difficulté d'interprétation du don via les encombrants et le caractère limité du Bookcrossing, certaines organisations ont mis en place des fêtes de la gratuité, sortes de marchés où chacun apporte (ou non) des objets dont il ne veut plus et repart avec ce qu'il veut (ou presque).

\footnotetext{
${ }^{10}$ Guillard V. et Roux D. (2014), Macromarketing Issues on the Sidewalk: How "Gleaners" and "Disposers" (Re)Create a Sustainable Economy, Journal of Macromarketing, 34, 3, 291-312.
} 


\section{Faire du don un événement}

Le Grand Don fait partie de ces initiatives qui se multiplient, en France et à l'étranger (en Italie notamment), autour du don d'objets dans l'espace public. Organisé à Paris sur le Pont Marie par des anonymes qui se présentent comme «la coordination permanente d'offrande publiques", cet évènement consiste en un "don collectif effectué par des inconnus à d'autres inconnus ». Il réunit tous ceux qui, sans contrepartie, viennent poser des objets sur le parapet du pont - sous réserve qu'ils soient transportables à pied - pour les offrir aux passants. L'absence de circulation monétaire fait que cette activité n'est pas répréhensible en soi - il n’y a pas d'actes de commerce dans l'espace public -, mais il n'y a pas non plus de commerce déguisé (en troc) parce que les objets n'ont qu'une très faible valeur marchande, comme le soulignent les organisateurs.

Le Grand Don ne se définit pas comme un évènement artistique ou caritatif mais comme « une expérience votive » lä̈que et citoyenne en place publique. En dépit du caractère apolitique affiché de la démarche, sa dimension militante est palpable au travers de sa volonté d'encourager une «logique du don gratuit» ou «du don pur» en marge du marché ${ }^{11}$. Les sources bibliographiques fournies en référence empruntent en effet de manière centrale aux penseurs de l'économie du don, de Godbout (2000) à Ricoeur $(2004)^{12}$, en soulignant le caractère «transgressif » et « transformateur» de ces pratiques.

Dans la lignée de ces évènements de don dans l'espace public, d'autres manifestations de troc et d'échanges de biens se développent parallèlement. Appelés «Really free free markets» (RRFMs) dans les pays anglo-saxons ${ }^{6}$, elles sont apparues en France sous le nom de gratiferias. Nées en Argentine en 2010, les gratiferias - littéralement des «fêtes de la gratuité » ou "marchés gratuits »- ont été initiées à Buenos Aires par Ariel Rodriguez Bosio, un jeune décroissant dont le projet a démarré par le don à des proches de biens qu'il jugeait superflus, puis par l'organisation de ces marchés dont la règle est: «Amenez ce que vous voulez, ou rien du tout. Repartez avec ce qui vous plait ». Rapidement populaires sur le continent américain, ces initiatives ont gagné l'Europe il y a peu. Faciles à organiser, que ce soit dans des lieux clos ou en plein air, dans des espaces publics ou privés, elles sont souvent prises en charge par des associations de quartiers ou des systèmes d'échanges locaux (S.E.L.) et s'appuient souvent sur l'aide des municipalités pour la fourniture d'un local ou l'occupation du domaine public.

Alors que le projet initial de leur fondateur était plutôt empreint d'une philosophie du détachement à l'égard des possessions matérielles - des objets devenus SPF ("Sans Propriétaire Fixe $»)^{7}-$, les gratiferias dévoilent l'abondance des objets dont les ménages n'ont plus l'utilité et dont ils souhaitent se débarrasser. Faisant suite à la mode des vide-greniers qui se sont développés depuis le début des années 1990, leur succès réside principalement dans le fait qu'elles instaurent une gratuité totale des échanges de biens dans un contexte économique parfois difficile pour certains. Ces marchés offrent de nouvelles modalités d'échanges entre particuliers qu'elles débarrassent de toute dimension marchande et calculatoire.

Hormis les bénéfices économiques qu'ils procurent à ceux qui viennent s'y approvisionner, ces évènements forment également des enclaves qui contribuent à diffuser l'esprit du don en marge du marché. Ils en constituent même le contrepoint en substituant à l'échange marchand un principe de réciprocité généralisée: celui qui donne n'attend rien en retour, mais il peut aussi prendre plus loin un objet qui lui est donné. Le caractère inhabituel du don d'objets à des inconnus entraîne ainsi des mécanismes de contre-dons qui se manifestent

\footnotetext{
${ }^{11} \mathrm{http}: / / g r a n d d o n . f r e e . f r / t e x t e s . h t m$

12 Godbout J. (2000), Le don, la dette et l'identité, Paris, La Découverte ; Ricoeur P. (2004), La lutte pour la reconnaissance et l'économie du don, conférence prononcée lors de la Journée de la philosophie (21 novembre 2002), Paris, Unesco.

${ }^{6}$ Albinsson P.A. et Perera B.Y. (2009), From trash to treasure and beyond: the meaning of voluntary disposition, Journal of Consumer Behaviour, 8, 6, 340-353.

7 Rousseaux A. (2012), «Zone de gratuité », ou comment les objets deviennent «sans propriétaire fixe » http://www.bastamag.net/Zone-de-gratuite-ou-comment-les, consulté le 20 mai 2015.
} 
moins par l'équation « un objet donné contre un objet récupéré ", mais par l'intérêt qu'un nouveau propriétaire lui porte à nouveau, la promesse qu'il fait d'en faire un bon usage, des remerciements, voire des marques de reconnaissance vis-à-vis de celui qui s'en débarrasse. Enfin, ces lieux créent à l'échelle locale des occasions de rencontre et participent à favoriser des liens sociaux, certes temporaires, mais plus riches que ceux qui se nouent de manière impersonnelle et anonyme sur Internet. Toutefois, ces évènements ne se pérennisent pas nécessairement dans le temps. Le dernier Grand Don date par exemple du 24 mai 2014 et les gratiferias sont encore des manifestations très ponctuelles dans la plupart des communes qui les ont organisées. Donner plus de régularité à ces systèmes d'échanges est l'objet d'autres initiatives comme les "arbres à livres ", les « give boxes» ou les « magasins pour rien ».

\section{Des lieux pour échanger?}

Les mairies, associations caritatives ou autres collectifs développent des lieux dédiés aux échanges gratuits. Ces lieux sont plus ou moins ouverts en termes d'accès sur la rue, ou encore en termes de catégories d'objets qui y circulent.

《Arbres 》, « armoires » ou «passages » à livres

Il s'agit de boîtes creusées dans des troncs d'arbres ou encore de meubles posés dans l'espace public, ou dans tout lieu destiné à accueillir les livres que les gens ne souhaitent plus conserver. Le principe des arbres à livres fait écho à celui d'une bibliothèque, mais sa particularité est d'être implanté dans la rue, ouvert et en autogestion. Les gens sont libres de prendre les ouvrages, de les déposer, de les lire sur place, de les reposer ou de les garder, afin de les faire circuler et de leur offrir une seconde vie.

Le principe d'un arbre à livres est proche du Bookcrossing déjà évoqué précédemment, mais contrairement à ce dernier, qui repose sur les plateformes Internet pour suivre la trace des livres, voire pour rencontrer leurs anciens possesseurs, l'arbre à livres est anonyme, installé de façon permanente dans un lieu fixe, et souvent à l'initiative conjointe des mairies et des associations afin, notamment, de promouvoir la culture pour tous.

Les arbres à livres s'inscrivent dans une conception de la vie de quartier où partage, échange et entraide contribuent à améliorer la qualité de vie, comme le sont les Givebox présentées ci-dessous, à la différence près que ces dernières ne sont pas restreintes à une seule catégorie d'objets.

\section{Giveboxes ou « armoires libres»}

Une Givebox (littéralement «boîte à donner ») est une grosse boîte, une armoire ou parfois un cabanon installé dans un quartier, dans une rue, ou plus rarement dans des lieux associatifs ou culturels par des collectifs de proximité ou autres associations caritatives. Ouverte $24 \mathrm{~h} / 24$, la Givebox est un dispositif de dons et/ou d'échanges gratuits d'objets, que ce soit des vêtements, des chaussures, des petits meubles, des appareils électroniques, etc. Popularisée en 2011 à Berlin, on en trouve aussi au Québec, en Grande-Bretagne, en Belgique et plus récemment en France. Pour que les riverains « jouent le jeu » et utilisent la Givebox pour faire circuler des objets, il est nécessaire qu'ils comprennent le sens, l'utilité, et ce qu'il est permis de faire avec (prendre et/déposer). Le don sans contrepartie n'étant pas une norme sociale encore largement répandue, certaines personnes peuvent avoir parfois - et surtout lors du premier usage - des réticences à l'utiliser. Elles doivent alors se l'approprier, lui trouver un intérêt, la respecter pour ne pas la considérer comme une « poubelle ». D'autant qu'il n'y a pas de « gardien » : elle est toujours ouverte et à la disposition de tous. De nombreuses animations et projets artistiques sont organisés autour des Giveboxes pour inviter les riverains à les découvrir et à en saisir l'utilité. 
Celle-ci est plurielle. Les objectifs d'une Givebox sont d'abord écologiques - les 11 premières boîtes en France ont permis l'échange de 108000 objets, soit 32 tonnes en une année 8 Leur mise en place a également une visée sociale : elle offre à tous un accès gratuit et illimité aux objets. Bien intégrée et appropriée par les riverains, elle peut devenir un objet identitaire, fédérateur et favoriser la rencontre et les interactions entre voisins. C'est pourquoi elle s'inscrit parfois dans un projet pédagogique et/ou artistique dans lequel étudiants en architecture et/ou en design mais aussi artistes, élus et bénévoles se rencontrent pour leur installation dans l'espace public comme ce fut le cas à Lyon fin 2014.

Les Giveboxes présentent toutefois une limite: elles n'ont qu'une capacité limitée de stockage et ne peuvent accueillir que des objets de faible encombrement. Un dernier dispositif offre alors la possibilité de faire circuler tous types d'objets : les « magasins pour rien ».

«Magasins pour rien » et « boutiques sans argent 》

Au 103 rue Vauban à Mulhouse, les personnes peuvent se rendre dans un "magasin pour rien ». Etymologiquement, le mot rien, issu du latin rem désigne une «chose ». Un " magasin pour rien » est alors un espace dans lequel on trouve des choses abandonnées (littéralement données à ban), autrement dit des choses laissées à la disposition d'autrui. Autrement appelés «boutiques sans argent», ces système de "dépôts-dons" permettent à chacun de donner et/ou prendre gratuitement toutes sortes d'objets (vêtements, objets de décorations, jeux de société, etc). La gérante fixe toutefois une limite : il n'est pas permis de prendre plus de trois objets par semaine, règle toutefois plus flexible pour les ménages en situation de (grande) pauvreté.

Le «magasin pour rien » de Mulhouse - le premier du genre en France - est le fruit d'une initiative locale, financée conjointement par la mairie et des associations de la «Maison de la citoyenneté mondiale de Mulhouse ». La gérante, payée sous contrat aidé subventionné par l'Etat, est assistée par des bénévoles qui rangent, nettoient, trient les objets apportés en toute discrétion par les habitants. Les frais de fonctionnement du magasin sont assurés par les dons des particuliers mais également par une subvention de la mairie.

Dans cette initiative, locale, le superflu des uns pourvoit aux besoins des autres. Ses objectifs sont pluriels : tout d'abord sociaux, ils s'inscrivent dans la mise en place d'actions pour lutter contre la pauvreté en offrant la possibilité, à ceux qui n'ont pas assez de moyens, d'accéder à des objets. Ensuite, le «magasin pour rien» renforce la solidarité entre ceux qui donnent et ceux qui reçoivent, sans pour autant que les premiers aient à affronter la situation précaire ou de pauvreté des seconds, et inversement sans que les seconds se sentent obligés de remercier ceux qui apportent leurs objets. Il est en effet difficile de donner des objets de la main à la main à ceux qui en ont besoin - si tant est que l'on connaisse leurs besoins - sans risquer de les humilier, de les gêner et de les endetter. Or il existe peu de structures où les actes de donner et de recevoir sont anonymes et lèvent les potentielles difficultés de mise en relation de ces deux populations. Les associations caritatives proposent des espaces anonymes de dons (des collecteurs placés à divers endroits dans les villes et généralement proches des lieux de passage ou de commerce), mais les dons collectés sont soit revendus dans des magasins caritatifs (Emmaüs par exemple ou encore Oxfam), soit recyclés lorsque leur qualité est insuffisante. Même si les prix des produits vendus dans les magasins caritatifs sont fortement décotés, ces lieux fonctionnent sur des bases monétaires et les objets continuent de circuler grâce à l'argent. Les «magasins pour rien» permettent au contraire de lever ces contraintes financières et relationnelles en rétablissant des mécanismes plus solidaires.

Cet espace gratuit poursuit un troisième objectif : écologique. En France, l'ADEME recensait en 2014, 325 millions de tonnes de déchets'. Parmi ces déchets qui arrivent en masse dans les centres de traitement, on trouve des objets qui sont encore en bon état de

\footnotetext{
${ }^{8}$ www.facebook.com/pages/Givebox-LYON/291157047749989?notif_t=fbpage_fan_invite

${ }^{9} \mathrm{http} / / / \mathrm{www}$.ademe.fr/chiffres-cles-dechets-edition-2014
} 
fonctionnement. Pour des raisons pratiques ou par négligence, ils sont parfois jetés avec les déchets ménagers ou encore déposés sur les trottoirs. Toutefois, s'ils ne sont pas ramassés par des glaneurs, ces objets finissent dans les déchetteries et entraînent des coûts de ramassage et de traitement. De fait, le «magasin pour rien » est précisément une solution pérenne qui facilite le sauvetage des objets et leur rencontre avec de nouveaux propriétaires.

Cette initiative locale essaime timidement en France : des bénévoles ont ouvert ce type de structure en Charente en 2014 ou encore aux Angles (Pyrénées Orientales) où un hangar permet aux uns de laisser des choses devenues inutiles pour que les autres viennent se servir. Un "magasin gratuit» a été ouvert au 88 rue de Ménilmontant à Paris, mais, faute de bonnes volontés pour le gérer, il oscille entre ouverture et fermeture par manque de régularité. La gratuité nécessite en effet de donner une autre ressource rare : du temps.

Toutes ces pratiques s'inscrivent pleinement dans la consommation collaborative, dans le sens où elles en réunissent les principaux ingrédients : une concentration de personnes souhaitant échanger, des modes de circulation des objets, des lieux où se déroulent ces échanges.

Au-delà d'un fourmillement de gestes et d'initiatives locales souvent peu médiatisés comparativement aux plateformes collaboratives, les dispositifs présentés remettent en question nos rapports à la consommation, mais aussi au temps, aux lieux et à autrui, comme aux formes dominantes de la nouvelle économie du partage.

Sur le plan de la consommation tout d'abord, ces dispositifs semblent changer le rapport des consommateurs à leurs possessions. Parce qu'ils ont la possibilité de les faire circuler plus facilement, les individus instaurent des relations avec leurs objets qui apparaissent plus liquides, moins fixes, moins centrales dans leur vie. Semblent, en apparence, car si certains se détachent davantage de leurs possessions qu'ils ont pléthore d'occasions de faire circuler, d'autres profitent de ce système pour accumuler ce qu'ils ne payent pas. Ils viennent dans ces lieux de gratuité par besoin de s'équiper parfois, mais bon nombre les fréquentent aussi pour le plaisir - régressif? de prendre. L'avidité et le plaisir de l'accumulation encouragés par la société de consommation demeurent présents dans ces systèmes d'échanges. Aussi, ces formes de redistribution ne contribuent pas encore complètement à ce que prônent les décroissants les plus militants : la sobriété volontaire, l'abolition du superflu ${ }^{10}$.

Sous cet angle, la gratuité apparaît comme un adjuvent de ces systèmes qui, elle aussi, offre un double sens de lecture. D'un côté, elle peut favoriser la boulimie d'objets ${ }^{11}$ et de l'autre aiguiser le regard porté sur les objets, non en fonction de leur prix, mais en fonction de leur valeur. Ainsi, ramasser, récupérer, reprendre un objet jeté ou donné oblige à s'interroger sur lui, à mobiliser des compétences techniques ou esthétiques, à faire sens de son sauvetage, et en tout cas à se comporter de manière moins passive que lorsqu'on achète un objet neuf dans un circuit conventionnel.

Ces formes gratuites de redistribution changent aussi le rapport au temps. Celui du capitalisme est celui de l'efficacité - atteindre un résultat -, voire de l'efficience - l'atteindre au moindre coût. On va dans un magasin pour trouver quelque chose de précis, on consulte Internet pour trouver rapidement le meilleur prix. A contrario, faire les encombrants, s'approvisionner dans l'espace public en récupérant un livre, un vêtement ou un bibelot dans une Givebox ou une gratiferia est affaire d'occasion, de hasard et parfois de rencontres. On prend le temps de voir, de s'arrêter, de flâner, de regarder, de réfléchir, voire de discuter. Ici règne le temps de l'imprévisibilité quand celui du commerce traditionnel est fait d'organisation, de régularités et de répétitions.

\footnotetext{
${ }^{10}$ Ariès P., (2011), La simplicité volontaire contre le mythe de l'abondance, La Découverte.

${ }^{11}$ Guillard V. (2014) (dir.), Boulimie d'objets ; L'Être et L'Avoir dans nos sociétés, Editeur : De Boeck.
} 
En termes de rapport aux lieux également, ces dispositifs de redistribution opèrent, pour certains, des changements notables par rapport aux formes conventionnelles d'échange. Hormis les «magasins pour rien », il n'y a plus de murs, plus de boutique. L'échange envahit l'espace public, qu'il s'approprie de manière temporaire et parfois détournée - en modifiant le sens que l'usage d'un pont (dans le Grand Don) ou d'un trottoir (pour les encombrants) ont dans l'imaginaire commun. Les modalités habituelles qui prévalent dans les lieux de commerce ou même dans les magasins de charité ne jouent plus: plus de vendeurs, de caisses, d'argent, de portiques de sécurité, d'attente pour payer. La mise en scène, qui n'est plus marchande, est réduite à son plus simple langage, c'est-à-dire à l'étalage des objets contraints à parler par et pour eux-mêmes.

En termes de rapports à autrui, il est désormais possible d'accéder à des objets de façon gratuite sans être pauvre (condition du don caritatif), sans être redevable (conséquence du don aux proches), sans devoir justifier d'un quelconque statut (de nécessiteux ou de client fidèle auquel on offre des cadeaux, etc.). Ces formes d'échange bousculent les normes sociales, voire l'étiquette des formes habituelles d'accès aux objets. Il faut s'autoriser à se servir de ce qui est laissé à la disposition de tous, apprendre à prendre sans contrepartie alors même qu'on aurait les moyens financiers d'acheter.

Enfin, ces systèmes de redistribution physiques non relayés par Internet questionnent les formes dominantes du modèle collaboratif. Alors que des interrogations émergent aujourd'hui sur les promesses de l'économie du partage, et sur leur capacité à marchandiser le travail des individus et leurs capitaux mobiliers et immobiliers ${ }^{12}$, les dispositifs que nous avons analysés demeurent ancrés dans une vision solidaire, locale et non capitalistique de la circulation des objets. Plus proches en cela de l'économie sociale que de l'exploitation des gisements financiers de l'économie peer-to-peer, ils semblent organiser à bas bruit une résistance de terrain ancrée sur la notion de gratuité.

De telles remises en cause des caractéristiques du capitalisme, dont certaines s’inscrivent pleinement dans le modèle de la décroissance, augurent d'une possible inflexion dans les manières de consommer et dans les valeurs dominantes d'une société sommée de penser en termes de soutenabilité : plus de solidarité et moins d'égoïsme ou d'individualisme ; plus de coopération que de compétition par l'argent ; plus de justice sociale et moins d'inégalités ; plus d'imprévu et moins d'obsession de la rentabilisation du temps ; plus d'attention portée aux objets et à leur durabilité ; plus de vie sociale et moins de plaisir marchand solitaire, etc.

Mais on peut s'interroger sur l'évolution future de ces dispositifs redistributifs. D'une part, leur volonté de pérennisation contribue à institutionnaliser ces pratiques, à les rendre moins hasardeuses et moins contingentes. D'autre part, "tout système non régulé faisant faillite ${ }^{13}$, ce mouvement d'institutionnalisation entraîne aussi des interrogations. Peut-on imaginer, par exemple, que les organisations marchandes s'immiscent dans la captation de valeur de ces flux d'objets usagés? Du côté des acteurs publics, les questionnements portent autant sur les problèmes de réduction des déchets dans les territoires, dont la réflexion est largement avancée, que sur les modes de régulation de ces activités dont les retombées sociales ou économiques sont pourtant loin d'être nulles : que doit-on réguler et comment, en termes d'occupation de l'espace public ? Faut-il contrôler ces échanges informels qui, s'ils demeurent marginaux, peuvent être amenés à prendre de l'ampleur? Jusqu'à quel point doit-on laisser ces initiatives se développer sans risquer de menacer l'ordre, y compris économique, qu'elles contestent silencieusement?

Valérie Guillard, Dominique Roux

\footnotetext{
${ }^{12}$ Dujarier M.-A. (2008), Le travail du consommateur : de MacDo à eBay, comment nous coproduisons ce que nous achetons, La Découverte, Paris; Schor J. (2014), Debating the sharing economy, Great Transition Initiative, http://greattransition.org/publication/debating-the-sharing-economy ; Dujarier

13 Thomas Piketty (2013), Le capital au XXIème siècle, Paris, Seuil.
} 
Valérie Guillard est maittre de conférences en marketing à l'université Paris-Dauphine, elle a récemment publié Boulimie d'objets, l'Etre et l'avoir dans nos sociétés (De Boeck, 2014).

Dominique Roux est professeur de marketing à l'Université Paris Sud et a notamment publié Marketing et résistance(s) des consommateurs (Economica, 2009). 